\title{
Calcium metabolism in sarcoidosis
}

\author{
C. E. DENT \\ Medical Unit, University College Hospital Medical School, London, W.C.1
}

\begin{abstract}
Summary
Fifteen patients with hypercalcaemia and sarcoidosis have been studied. Twelve were males of average age 34; the three females averaged 53.

Cases included widely varying sarcoid manifestations, but five gave a clear history of rather excessive ingestion of low-dosage vitamin D preparations. The data confirm that in most cases there is an undue sensitivity to all the actions of vitamin $D$, the situation therefore mimicking vitamin $D$ intoxication. Two patients volunteered to receive ultra-violet irradiation and became hypercalcaemic with corresponding clinical and biochemical changes.

Steroids make normal the calcium abnormalities just as they do in straight vitamin $D$ intoxication. However, in three further patients the hypercalcaemia did not respond to steroids and was shown to be due to the presence of an over-acting parathyroid gland, removal of which corrected the abnormality. There are a sufficient number of other similar cases in the literature to suggest that the development of parathyroid adenomas is another even rarer complication of sarcoidosis which must be carefully distinguished from vitamin $D$ sensitivity.
\end{abstract}

It has been known for some time that certain patients with sarcoidosis also manifest a disorder of calcium metabolism. This was discovered partly because they used to be treated with large doses of calciferol and it was obvious from the rapid intoxication which sometimes ensued that they could be unduly sensitive to this vitamin. It was then discovered that some manifested hypercalcaemia apparently spontaneously. Finally some of these patients presented with urinary stones and hypercalcuria, others with renal failure, which could not be explained as the result of involvement of the kidney by the sarcoid granulomas. These latter situations gave grounds for the suspicion that hypercalcaemia had been present previously even if not found to be present still. This was made even more likely by the occurrence in some of corneal calcification, a sign possibly pathognomonic of past or present hypercalcaemia.

My own interest in the problem arose from the need to distinguish such hypercalcaemic sarcoid patients from those with hypercalcaemia of other origins. The problem was early highlighted by a mis-diagnosis I made in 1950 of primary hyperparathyroidism in a patient who really had sarcoidosis. This latter patient manifested no clinical signs of sarcoidosis at the time of diagnosis although she developed a typical rash some years after I had advised her to have a neck exploration which of course was unsuccessful. This problem of differential diagnosis was not much helped by purely clinical studies. Some patients with primary hyperparathyroidism manifested remarkably few specific clinical features. Patients with hypercalcaemia as a complication of sarcoidosis did not belong to any particular clinical type. The only small help came from a study of their age distribution which in a few cases tended to be lower than usual, of their sex which was predominantly male, and from the fact that a high proportion had been taking vitamin D for some reason or other in doses that would not produce hypercalcaemia in a normal patient. I now summarize some data for our fifteen hypercalcaemic sarcoid patients, which includes that of our first five published already (Dent, 1958): ten had corneal calcification, one iridocyclitis, five lymphadenopathy, five splenomegaly, nine miliary chest shadowing, one osteitis cystoides multiplex, six various rashes, five gave a clear history of self medication with vitamin $D$ preparations. There were twelve males and three females, the average age 34 years in the males (who included the three youngest of 14,15 and 23 years), 53 years in the females. The maintenance dose of steroid on follow up was 25$62.5 \mathrm{mg}$ of cortisone, or $40 \mathrm{mg}$ of hydrocortisone or, in one case, $6 \mathrm{mg}$ of prednisone.

We experimented very early with the use of cortisone as a means of differential diagnosis and it soon became clear that patients with hypercalcaemic sarcoidosis responded well to cortisone at a dose of $50 \mathrm{mg}$ 8-hourly for 10 days, the plasma calcium falling, often to normal, during this time (Fig. 1) and it could be kept normal with a much diminished dose, sometimes of the order of $25-50 \mathrm{mg}$ /day such as is only useful otherwise in the maintenance of Addison's disease (see above). Nevertheless on this 


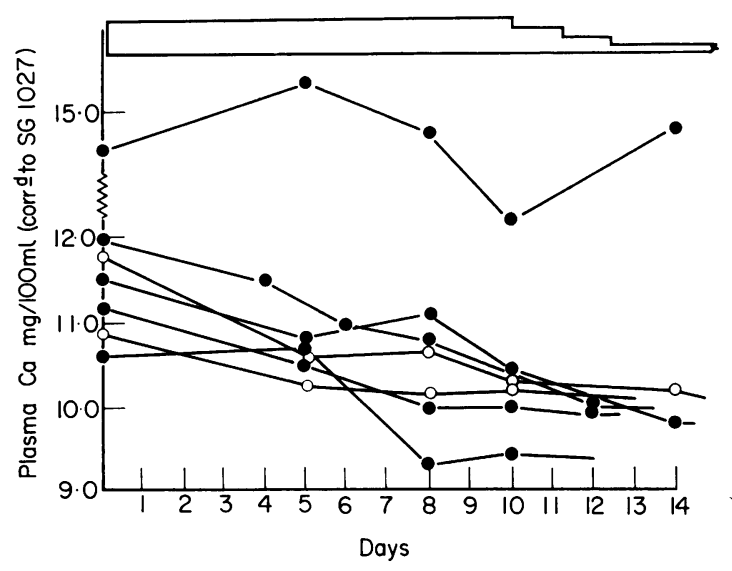

FIG. 1. Response of hypercalcaemic sarcoidosis patients to standard test with cortisone or hydrocortisone. Six patients showed a typical response, namely, a rapid fall to normal in plasma calcium level. The seventh who maintained very high fluctuating levels was later shown to have a parathyroid adenoma, removal of which led to a fall to normal of his plasma calcium (Dent \& Watson, 1966). $\mathrm{O}=$ Cortisone $50 \mathrm{mg} 8$-hourly, Hydrocortisone $40 \mathrm{mg} 8$-hourly.

small dose most of the sarcoid lesions also disappeared in the next few months as if to suggest that the lesions were particularly sensitive in these patients. In primary hyperparathyroidism the hypercalcaemia was not thus affected (Dent \& Watson, 1968).

Further studies of this calcium abnormality at U.C.H. (Anderson et al., 1954) and independently by Henneman and his group in the U.S.A. (Henneman et al., 1956) showed that the hypercalcaemic state in sarcoidosis closely resembled that of vitamin D intoxication. Furthermore some patients with sarcoidosis without hypercalcaemia could be induced to develop this hypercalcaemic state by rather small doses of vitamin D. These observations helped to explain another early clinical finding which was difficult to investigate fully namely that some of these patients with sarcoidosis became ill when exposed to bright sunlight. We have had the opportunity of irradiating with artificial UV light two patients (Figs. 2 and 3) who volunteered to test this effect, one under full calcium balance control. It can be seen that both these patients became hypercalcaemic with the irradiation. The balance changes (Fig. 3) resembled those of vitamin D intoxication, exactly as would be obtained in a normal person given very large doses of vitamin $D$. Of even more interest was the fact that giving cortisone reversed all these metabolic changes even though the irradiation was being continued. Hence we think we have proved that there is a true sensitivity to vitamin D taken either orally or from sunlight in these particular

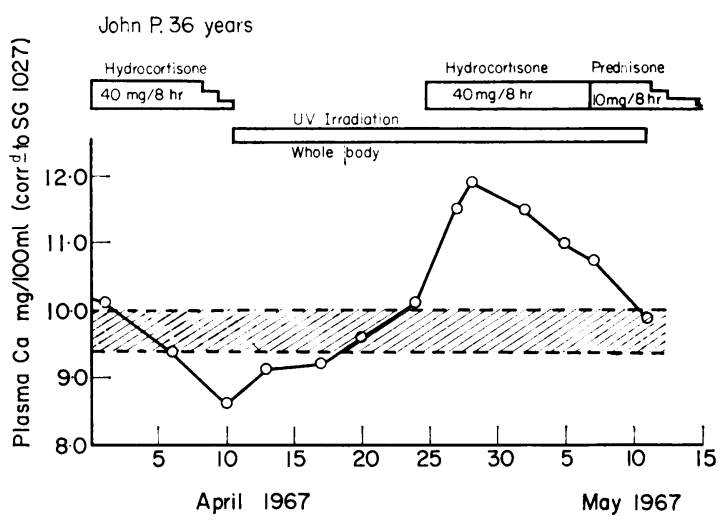

FIG. 2. Effects of UV and corticosteroid on patient with sarcoidosis. This patient had some months previously taken small doses of vitamin D and was slightly hypercalcaemic on admission. The first course of steroid lowered this, it was then raised with UV irradiation, and again lowered, the UV being continued, when steroid was again given. The dose of vitamin $\mathbf{D}$ thus given, while effectively antirachitic in normals. is far too small to produce hypercalcaemia in a normal person.

patients with sarcoidosis and that this is in some way antagonized by cortisone. This result, by the way, led to the investigation of the effect of cortisone in ordinary vitamin $D$ intoxication in patients with other diseases. It was found to be quickly effective in reversing all the signs and symptoms, in other words, cortisone is an antidote to vitamin $\mathrm{D}$ intoxication.

We are still puzzled as to the possible mechanism by which the chronic sarcoid granuloma sometimes produces this sensitization to vitamin $\mathrm{D}$, but current work on its metabolism suggesıs possible explanations. We now know that vitamin $D$ has to be converted to 25-hydroxycholecalciferol before it can exert its usual action (Blunt, De Luca \& Schnoes, 1968). In some cases of so called 'vitamin D resistance' (for instance in renal failure) this conversion is somehow inhibited (Avioli et al., 1968). One only has to postulate that in the relevant patients with sarcoidosis the conversion is facilitated, a theory which is open to experimental verification by existing methods. This theory might also explain another of our clinical observations, namely, that sarcoid patients who develop chronic uraemia do not seem to get renal osteodystrophy.

We have used the cortisone test in all our fifteen patients with hypercalcaemic sarcoidosis. They all responded well except one who had marked liver involvement. He responded better to hydrocortisone, perhaps because his liver was incapable of converting the cortisone given to the active hydrocortisone compound. We therefore now use hydrocortisone instead of cortisone for our standard test in the 
Michael L.

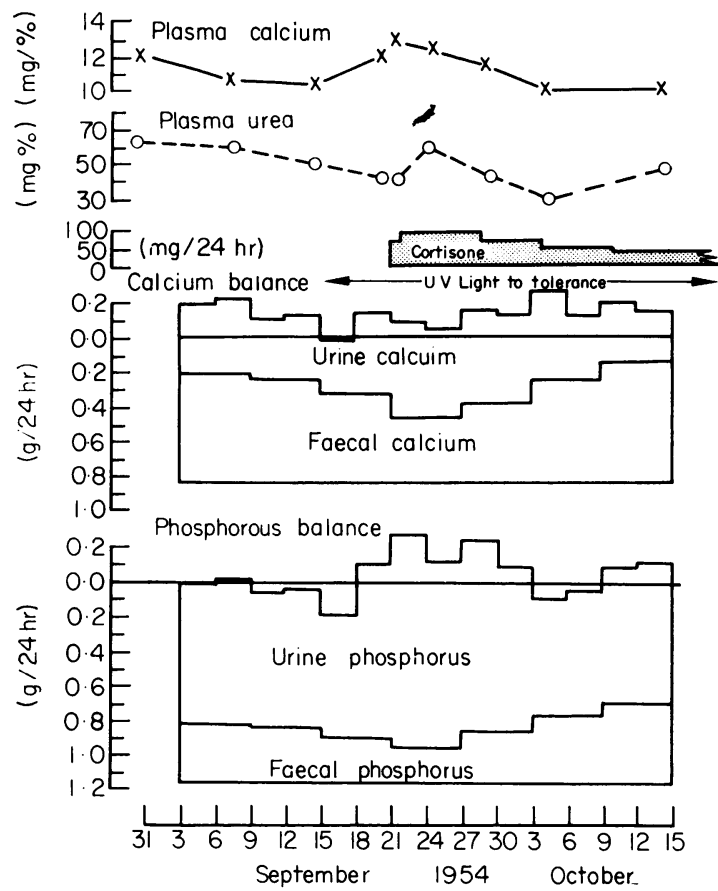

FIG. 3. Data from patient with hypercalcaemic sarcoidosis. His control balance already shows some signs of mild vitamin D overdose: high urinary calcium, hypercalcaemia and negative calcium balance. On UV irradiation the plasma calcium rose with increase in hypercalciuria and fall in faecal calcium, the balance being hardly changed. The addition of cortisone reversed these changes in spite of continuing the UV irradiation. The changing calcaemia without corresponding changes in external balance show the 'parathormone-like' effect of the vitamin $D$ intoxication in this sensitive patient, that is, a change in equilibrium between the calcium in bone and in extra-cellular fluids.

(Presented at the VIIIth Middle East Medical Assembly, Beirut, 11 May 1958 and reproduced by permission.)

differential diagnosis of hypercalcaemia using the equivalent dose of $40 \mathrm{mg}$ 8-hourly for 10 days (Dent \& Watson, 1968).
The differential diagnosis may however be more difficult than this for we have had three cases of our own and have seen several elsewhere and in the literature in which patients with sarcoidosis identified by biopsy and other means had a hypercalcaemia that was not responsive to hydrocortisone (one is shown in Fig. 2). All these patients were found to have parathyroid adenomas as well, which when removed dealt with the hypercalcaemia adequately, thus showing that the adenoma was the entire cause in these patients. I believe that finding three such patients in our series is likely to be more than a coincidence and am speculating that sarcoidosis may sometimes stimulate the parathyroid glands by an unknown mechanism to produce adenomas. If this is confirmed there will be yet another metabolic complication to add to that of the vitamin D sensitivity and it is interesting to wonder if there may be some link between these two.

\section{Acknowledgment}

The author wishes to thank Dr P. Heaf for permission to use data from his patient shown in Fig. 2.

\section{References}

Anderson, J., Dent, C.E., Harper, C. \& Philpot, G.R. (1954) Effect of cortisone on calcium metabolism in sarcoidosis with hypercalcaemia. Possibly antagonistic actions of cortisone and vitamin D. Lancet, ii, 720.

Avioli, L.V., Birge, S., Lee, S.E. \& Slatopolsky, E. (1968) The metabolic fate of vitamin $\mathrm{D} 3-3 \mathrm{H}$ in chronic renal failure. Journal of Clinical Investigation, 47, 2239.

Blunt, J.W., De LuCA, H.F. \& Schnoes, H.K. (1968) 25hydroxycholecalciferol. A biologically active metabolite of vitamin D3. Biochemistry, 7, 3317.

Dent, C.E. (1958) Proceedings of the 8th Middle East Medical Assembly, Beirut, p. 162

DenT, C.E. \& WATSON, L. (1966) Hyperparathyroidism and sarcoidosis. British Medical Journal, i, 646-649.

DENT, C.E. \& WATSON, L. (1968) The hydrocortisone test in primary and tertiary hyperparathyroidism. Lancet, ii, 662.

Henneman, P.H., Dempsey, E.F., Carroll, E.L. \& Albright F. (1956) The cause of hypercalciuria in sarcoid and its treatment with cortisone and sodium phytate. Journal of Clinical Investigation, 35, 1229-42. 